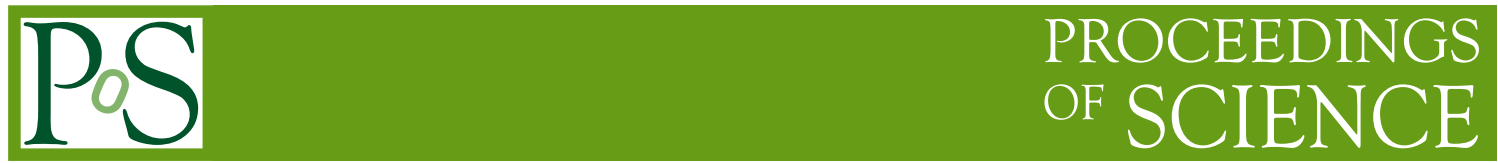

\title{
NuSTAR Observations of X-Ray Binaries
}

\section{John A. Tomsick ${ }^{* \dagger}$}

Space Sciences Laboratory, 7 Gauss Way, University of California, Berkeley, CA 94720-7450, USA

E-mail: jtomsickessl.berkeley.edu

Eric Bellm, Felix Fuerst, Fiona Harrison, Hiromasa Miyasaka, Shriharsh Tendulkar

Cahill Center for Astronomy and Astrophysics, Caltech, Pasadena, CA 91125, USA

E-mail: fionalsrl.caltech.edu

\section{Varun Bhalerao}

Inter University Center for Astronomy and Astrophysics, P.O. Bag 4, Ganeshkhind, Pune

411007, India

E-mail: varunb@iucaa.ernet.in

\section{Deepto Chakrabarty}

Kavli Institute for Astrophysics and Space Research, MIT, 70 Vassar St., Cambridge, MA 02139,

USA

E-mail: deepto@mit.edu

\section{Ashley King}

Department of Physics, 382 Via Pueblo Mall, Stanford, CA 94305, USA

E-mail: ashking@stanford.edu

Jon M. Miller

Department of Astronomy, University of Michigan, 1085 S. University Ave., Ann Arbor, MI 48109-1107, USA

E-mail: jonmm@umich.edu

\section{Lorenzo Natalucci}

Istituto Nazionale di Astrofisica, INAF-IAPS, via del Fosso del Cavaliere, I-00133, Roma, Italy

E-mail: Lorenzo.Nataluccieiaps.inaf.it

\section{Daniel Stern}

Jet Propulsion Laboratory, Caltech, Pasadena, CA 91109, USA

E-mail: daniel.k.sternejpl.nasa.gov 


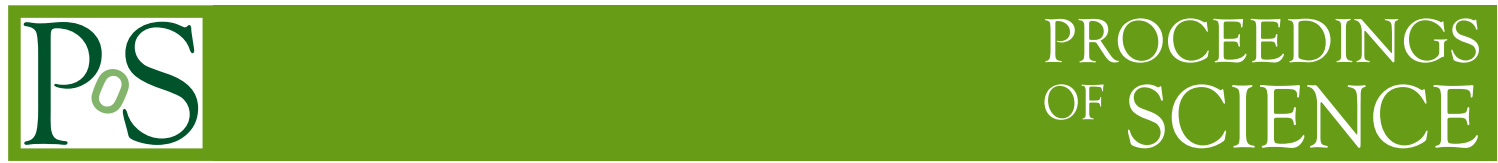

As of 2014 August, the Nuclear Spectroscopic Telescope Array (NuSTAR) had observed $\approx 30 \mathrm{X}$ ray binaries either as part of the planned program, as targets of opportunity, or for instrument calibration. The main science goals for the observations include probing the inner part of the accretion disk and constraining black hole spins via reflection components, providing the first observations of hard X-ray emission from quiescent Low Mass X-ray Binaries (LMXBs), measuring cyclotron lines from accreting pulsars, and studying type I X-ray bursts from neutron stars. Here, we describe the science objectives in more depth and give an overview of the NuSTAR observations that have been carried out to achieve the objectives. These include observation of four "IGR” High Mass X-ray Binaries (HMXBs) discovered by INTEGRAL. We also summarize the results that have been obtained and their implications. Among the IGR HMXBs, we focus on the discovery of a cyclotron line in the spectrum of IGR J17544-2619.

10th INTEGRAL Workshop: "A Synergistic View of the High Energy Sky" - Integral2014, 15-19 September 2014

Annapolis, MD, USA

\footnotetext{
${ }^{*}$ Speaker.

$\dagger$ This talk was given on behalf of the NUSTAR Galactic Binaries Working Group.

†Einstein Fellow
} 


\section{Introduction}

The NUSTAR satellite launched in 2012 June, and has been carrying out science operations for almost 2 years (at the time of the conference). NuSTAR observes in the $3-79 \mathrm{keV}$ bandpass and is the first focusing hard X-ray telescope in orbit. Its sensitivity, angular resolution (58" HalfPower Diameter), and energy resolution above $10 \mathrm{keV}$ are unprecedented (Harrison et al., 2013). NUSTAR's field of view is $12 \times 12 \mathrm{arcmin}^{2}$, which precludes an all-sky survey. Thus, the standard NUSTAR operation is to point from source-to-source, and a detailed observing plan is required.

The mission has broad science goals across many areas of astrophysics. The good angular and energy resolution in the hard X-ray band ( $900 \mathrm{eV}$ FWHM at $60 \mathrm{keV}$ ) allows for a sensitive study of ${ }^{44} \mathrm{Ti}$ emission lines from supernova remnants like Cas A (Grefenstette et al., 2014). The sensitivity, angular resolution, and timing capabilities have also been used to find and identify stellar remnants near the Galactic center (Mori et al., 2013) and in the Galactic plane (Gotthelf et al., 2014). NUSTAR's sensitivity is extending our reach into the Universe by detecting fainter Active Galactic Nuclei (Alexander et al., 2013).

Here we report on the NUSTAR program for observing X-ray binaries in the Galaxy. Section 2 introduces the topics and science objectives that we used in guiding our observing program. Section 3 summarizes the observations that had been carried out as of 2014 August. Section 4 summarizes results to date, including follow-up observations of the intermediate luminosity sources discovered by INTEGRAL.

\section{Science Objectives for X-ray Binaries}

In designing the X-ray binaries program, the Galactic Binaries Working Group has considered the full array of NUSTAR capabilities. In considering the most important advances that NUSTAR provides, we divided our program into the following four topics:

1. Reflection from black hole accretion disks: A Compton reflection component is seen when a hard X-ray source illuminates the accretion disk, and this produces fluorescent iron line emission, iron absorption edges, and a hard X-ray reflection hump (Lightman \& White, 1988; Fabian et al., 1989). These features are distorted by Doppler and gravitational effects when the disk approaches the black hole, and allows for a measurement of the geometry of the source and the accretion disk. NUSTAR's ability to do this measurement was demonstrated early-on in the mission for AGN (Risaliti et al., 2013). Once it was clear that the NuSTAR bandpass would extend down to $3 \mathrm{keV}$, we saw that this would allow for a measurement of this full reflection component, and several observations of black holes and neutron stars have been carried out with a reflection measurement as a goal (see below). The targets have been mostly bright sources (e.g., Cyg X1, GRS 1915+105, Ser X-1), and the throughput of NuSTAR's CZT detectors has been of critical importance.

2. Quiescent Low Mass X-ray Binaries: While X-ray binaries are bright when they are in outburst, most accreting black holes and many accreting neutron stars are transient, and become very faint when they are in quiescence. With Chandra and XMM-Newton, it became possible to detect the majority of the quiescent transients and to study several in detail in the soft X-ray band, 
no previous satellite could observe them in the hard X-rays. With NuSTAR's sensitivity, we have been able to observe some of these systems for the first time.

3. High Mass X-ray Binaries and accreting pulsars: For most of the known High Mass $\mathrm{X}$-ray Binaries (HMXBs), the accreting compact object is a neutron star with a relatively high magnetic field. These systems often exhibit pulsations, they sometimes show Cyclotron Resonant Scattering Features or "cyclotron lines" (Truemper et al., 1978; Coburn et al., 2002), and they typically have a continuum that is very hard, peaking near $15-25 \mathrm{keV}$ with a high-energy cutoff. The cyclotron lines are dips in the energy spectrum at energies which are directly and linearly related to the neutron star magnetic field. A magnetic field strength of $10^{12} \mathrm{G}$ corresponds to a line at $12 \mathrm{keV}$, placing line energies for most HMXBs in the middle of the NuSTAR bandpass. With NUSTAR's energy resolution and sensitivity, more detailed studies of cyclotron lines are possible, and they are detectable for more of the fainter systems. As INTEGRAL has led to a large increase in the HMXB population, and many of the "IGR" HMXBs have lower fluxes, an important part of our program is to study IGR HMXBs.

4. Type I X-ray bursts: Thermonuclear X-ray flashes occur in systems where accretion onto a low magnetic field $\left(\sim 10^{8-9} \mathrm{G}\right)$ neutron star occurs. Typically, the burst spectrum is a $\sim 1-3 \mathrm{keV}$ blackbody where the temperature evolves while the flux changes by orders of magnitude over a time period of $\sim 10-100$ s (Lewin, van Paradijs \& Taam, 1993). Some systems show bursts that are powerful enough to cause expansion of the neutron star's photosphere, and a fraction of these are "superexpansion" bursts where the photosphere increases its radius by a factor of $\sim 100$. It is in these superexpansion bursts where absorption edges may be present. With the energy resolution of the Rossi X-ray Timing Explorer Proportional Counter Array, these features appear as maxima in the residuals near 6-7 keV and minima near 10-13 keV (in't Zand \& Weinberg, 2010), and there are heavy elements ( $\mathrm{Fe}, \mathrm{Co}, \mathrm{Zn}, \mathrm{Ni}$ ), which have absorption edges that could cause the observed features. With the improvement in energy resolution, NUSTAR will be able to confirm the nature of these features and measure the edge energies.

\section{Overview of NuSTAR Observations}

For most of the NuSTAR observations carried out through 2014 August, we have extracted count rates in the 4-6 keV, 6-12 keV, and 12-25 keV energy bands, and Figure 1 shows a hardnessintensity diagram where each point represents a individual NuSTAR observation. There are rates from 33 X-ray binaries shown on the plot, and most of them have been observed one or two times. The sources that have been most frequently observed are the accreting black holes Cyg X-1 and GX 339-4. The exposure times are typically $\sim 30 \mathrm{ks}$ per observation, but they range from a few ks (especially for some of the observations during in-orbit checkout) to $\sim 100 \mathrm{ks}$ for the quiescent LMXBs.

Figure 1 gives some indication of how much time we have spent on each of our science objectives outlines above. Most of the black hole observations are related to Goal \#1 (reflection). For Goal \#2 (quiescent LMXBs), we have observed Cen X-4 and V404 Cyg, and these are the brightest of the quiescent LMXBs. We have observed several accreting pulsars for Goal \#3 (cyclotron lines), and some bursters for Goal \#4 (absorption edges). 


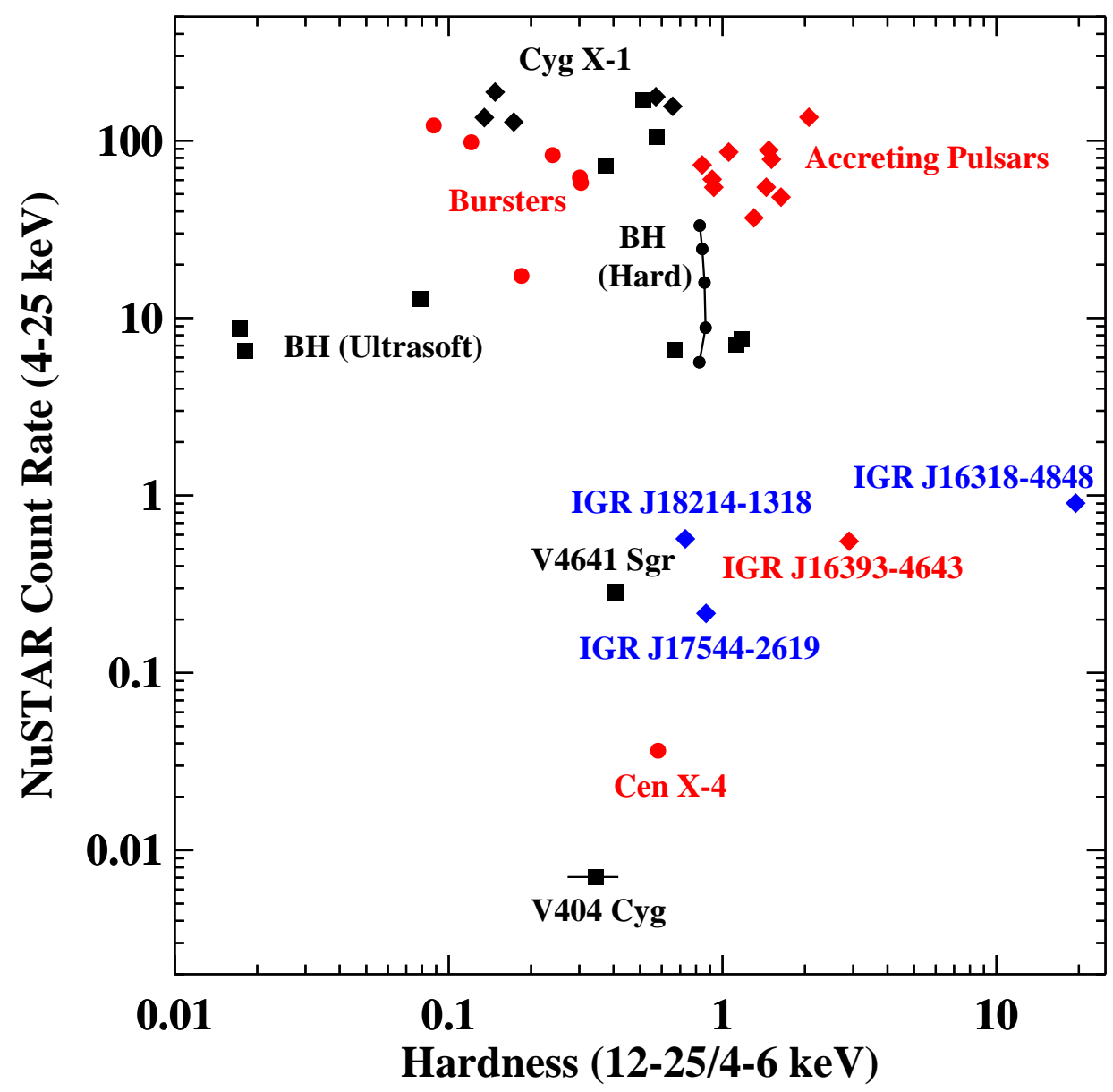

Figure 1: Hardness-intensity diagram for X-ray binaries (black points are accreting black holes and red points are accreting neutron stars) observed by NUSTAR through 2014 August. The black diamonds are observations of Cyg X-1 in the hard and soft state, and the five connected circles are observations of GX 339 4 in the hard state.

The observations take advantage of the very large dynamic range of NUSTAR, which can make useful observations of sources with count rates extending over four orders of magnitude. The limit at the high count rate end is caused by deadtime, but, even for a source as bright as Cyg X-1, the NUSTAR deadtime is much lower than a CCD instrument (Tomsick et al., 2014), there is no significant pile-up, and the spectrum is not distorted. The low count rate limit depends on the science goals. Our goals at least involved measuring the energy spectrum and obtaining a detection above $10 \mathrm{keV}$, and for this, the V404 Cyg rate is close to the limit for a $\sim 100 \mathrm{ks}$ observation. The location of the four IGR HMXBs that we have observed are at intermediate count rates on the hard side of the plot. This is a region of parameter space where NUSTAR exposures of moderate duration can make a huge improvement over previous measurements, and we provide a more detailed example below. 


\section{Results and Discussion}

Here, we provide a brief status report on the results that have been obtained for each of the four goals listed above. Several papers are in preparation, but we restrict ourselves to describing published work.

\subsection{Goal \#1: Reflection}

There are three black hole systems for which we have used modeling of the reflection component to constrain the spin of the black hole: Cyg X-1, GRS 1915+105, and 4U 1630-47. For Cyg X-1, the study used $30 \mathrm{ks}$ NuSTAR and Suzaku observations from 2012 October 31 - November 1, covering the 1-300 keV bandpass (Tomsick et al., 2014). The source was in the soft state, and the spectrum includes a multi-temperature blackbody, a power-law, and a strong reflection component. The Fe $\mathrm{K} \alpha$ emission line is very broad and the line and reflection component are consistent with the relativistic smearing expected if the emission is coming from the inner accretion disk. Assuming that the disk is at the innermost stable circular orbit (ISCO), we obtained a black hole spin of $a_{*}>0.83$ (Tomsick et al., 2014), which is consistent with previous measurements using the reflection component (Duro et al., 2011; Miller et al., 2012; Fabian et al., 2012) and using a method of modeling the thermal continuum (Gou et al., 2014). The reflection modeling also provides a constraint on the inclination of the inner disk, and we find that $i>40^{\circ}$ (Tomsick et al., 2014), which is significantly higher than the value of $i=27.1^{\circ} \pm 0.8^{\circ}$ obtained for the binary inclination (Orosz et al., 2011). This may indicate that there is a warp in the accretion disk.

We also used reflection modeling to measure the black hole spins for GRS $1915+105$ and $4 \mathrm{U}$ 1630-47. For GRS 1915+105, the NuSTAR measurement with $1-\sigma$ statistical errors is $a_{*}=$ $0.98 \pm 0.01$ (Miller et al., 2013b), and this compares well with the previous value found with thermal modeling (McClintock et al., 2006). For 4U 1630-47, the value (also with 1- $\sigma$ statistical errors) is $a_{*}=0.985_{-0.014}^{+0.005}$ (King et al., 2014), and this is the first time a black hole spin measurement has been obtained for this system. In addition to the broad emission line, absorption was also seen in the system, and King et al. (2014) interpret this as a blue-shifted absorption line from a high-velocity disk wind.

We obtained NUSTAR observations of 1E 1740.7-2942, which is an accreting black hole and "microquasar" (an X-ray binary with radio jets) within $\approx 1^{\circ}$ of the Galactic Center. The source was in the hard state when we observed it, which is its most common state. As the source is in a very crowded region, NUSTAR provides an advance since we are able to definitively obtain an uncontaminated hard X-ray spectrum. We measured a spectrum that is well-described by thermal Comptonization, and we did not detect a reflection component with $90 \%$ confidence upper limits on the covering fraction of 1-8\%, depending on the disk ionization (Natalucci et al., 2014).

In addition to the black hole results, we measured the reflection component for the neutron star LMXB Ser X-1 with NUSTAR, marking the first time that the hard X-ray reflection hump was detected in a neutron star system (Miller et al., 2013a). As the inner disk edge is close to the neutron star surface, such measurements may be promising for obtaining a constraint on the neutron star radius. 


\subsection{Goal \#2: Quiescent LMXBs}

NuSTAR observed the neutron star LMXB Cen X-4 for 114 ks in 2013 January. Part of the observation was also covered by XMM-Newton, and the results were reported in Chakrabarty et al. (2014). Previous observations with XMM-Newton and other soft X-ray satellites of Cen X-4 in quiescence show that the spectrum has thermal emission with a temperature of $\sim 0.1 \mathrm{keV}$ from the neutron star surface as well as a hard component that is consistent with being a power-law with an index of $\Gamma=1-2$ (Cackett et al., 2010). The power-law component has never been detected above $10 \mathrm{keV}$, and a major question has been where the cutoff to the component occurs. The NuSTAR observation shows a spectrum that can be fitted with an exponential cutoff at $10.4 \pm 1.4 \mathrm{keV}(1-\sigma$ uncertainties), and we found that the hard component can also be explained as $k T=18.2 \pm 1.0 \mathrm{keV}$ bremsstrahlung emission (Chakrabarty et al., 2014). We considered the physical implications of the hard component originating from inverse Compton, synchrotron shock, and bremsstrahlung mechanisms and found that inverse Compton and synchrotron shock are ruled out on physical grounds. We favor a scenario where the hard component is bremsstrahlung emission from a hot layer above the neutron star atmosphere (Chakrabarty et al., 2014; D'Angelo et al., 2014).

\subsection{Goal \#3: Accreting Pulsars and Cyclotron Lines}

Thus far, NUSTAR measurements of cyclotron lines have been reported for the accreting pulsars Her X-1 (Fürst et al., 2013), Vela X-1 (Fürst et al., 2014b), KS 1947+300 (Fürst et al., 2014a), and RX J0520.5-6932 (Tendulkar et al., 2014). While they all have relatively typical magnetic field strengths of a few times $10^{12}$ G, Suzaku reported that GRO J1008-57 has a cyclotron line near $80 \mathrm{keV}$ (Yamamoto et al., 2014), and a combined NuSTAR and Suzaku study confirmed the presence

of a line at $78_{-2}^{+3} \mathrm{keV}$ (Bellm et al., 2014). We also used the NuSTAR spectrum to look for the possibility of a fundamental cyclotron line at lower energies (e.g., if the $78 \mathrm{keV}$ line is a harmonic, then we might find a fundamental near $39 \mathrm{keV}$ ). However, we did not find any evidence for a line at lower energy, providing strong evidence that the $78 \mathrm{keV}$ line is the fundamental, indicating a neutron star magnetic field strength near $7 \times 10^{12} \mathrm{G}$ (Bellm et al., 2014).

A NuSTAR observation of the HMXB IGR J17544-2619 led to the first detection of a cyclotron line in a Supergiant Fast X-ray Transient (SFXT). SFXTs have unusually bright flares, and it has been suspected that these might be related to very strong neutron star magnetic field strengths (Bozzo, Falanga \& Stella, 2008). However, the line energy is $16.8 \pm 0.3 \mathrm{keV}$ (Bhalerao et al., 2014), which corresponds to a typical value for a neutron star HMXB. Figure 2 shows the NuSTAR and Swift/XRT spectra from an observation that occurred on 2013 June 18-19 fitted with a $\sim 1 \mathrm{keV}$ blackbody, a power-law with an exponential cutoff, and a cyclotron line. More than one continuum model can provide a good fit to the spectrum, but the cyclotron line is always required. Simulations were performed to determine that the statistical significance of the line is $>5-\sigma$. The spin period of the IGR J17544-2619 neutron star is still unclear, but knowing this would be very helpful in understanding the production of the large flares from SFXTs.

Although the NUSTAR observation of GS 0834-430 did not lead to a cyclotron line detection, we studied the energy dependence of the $12.3 \mathrm{~s}$ pulsations from this accreting pulsar. The pulse profiles show a very strong hard phase lag with a shift by 0.3 cycles going across the NuSTAR bandpass (Miyasaka et al., 2013). This was the first time that such large energy-dependent lags have 


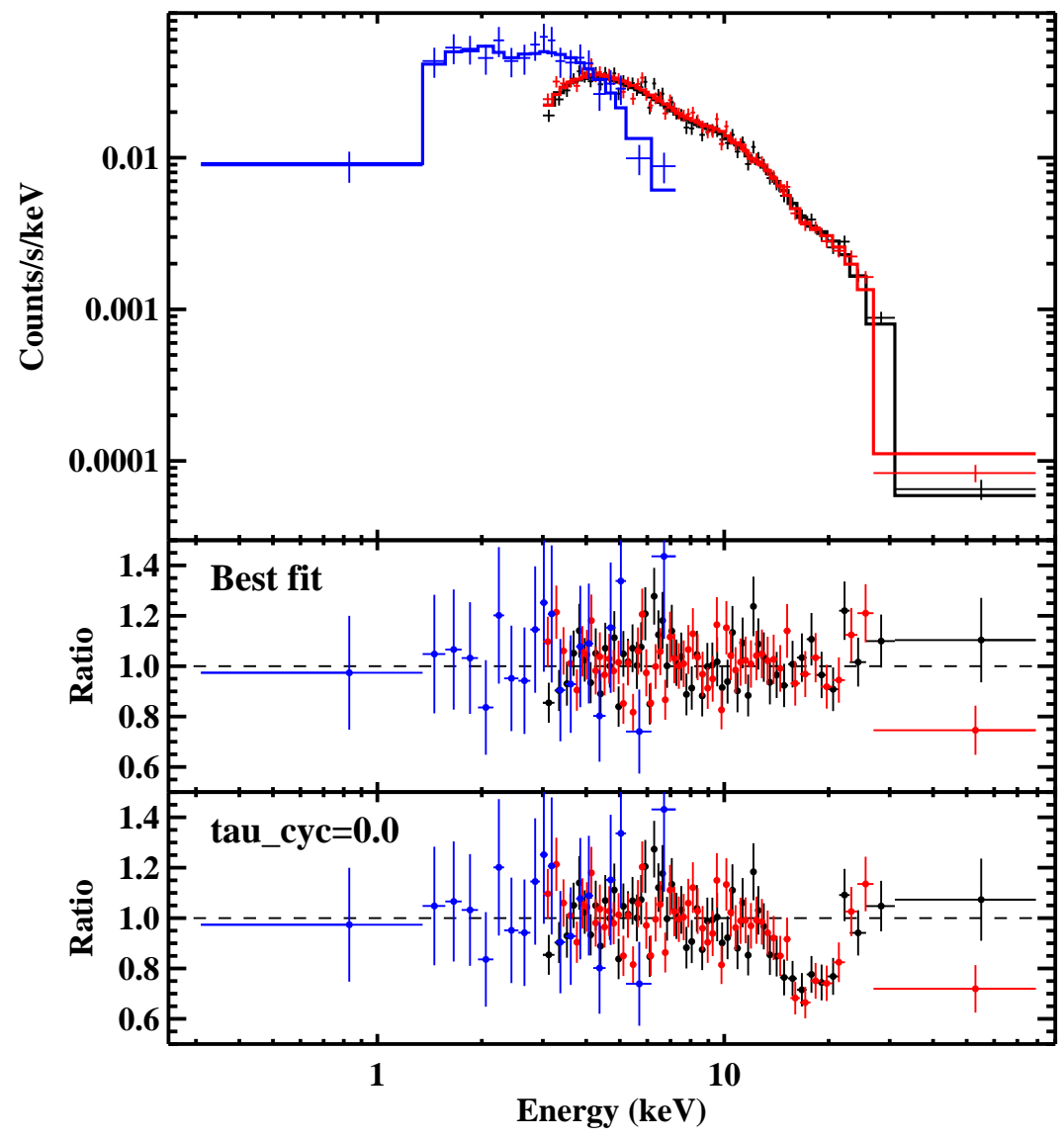

Figure 2: Swift/XRT and NuSTAR spectrum of the Supergiant Fast X-ray Transient IGR J17544-2619. The observations occurred on 2013 June 18-19, and the NuSTAR exposure time was 27 ks (Bhalerao et al., 2014). The top panel shows the count spectrum fitted with a blackbody, a cutoff power-law, and a cyclotron line. The middle panel shows the residuals to that model, and the bottom panel shows the residuals when the cyclotron line optical depth is set to zero.

been reported for an HMXB, and Miyasaka et al. (2013) conclude that the most likely explanation for the lags is a complex pulsar beam geometry.

\subsection{Goal \#4: Type I X-ray Bursts}

For our observing program, we selected bursters that are known to enter states where bursts occur frequently (e.g., every few hours) to provide a good chance that NUSTAR will detect bursts. Furthermore, with detection of absorption edges during superexpansion bursts being our primary goal, we selected Ultracompact X-ray Binaries (UCXBs) since they are known to be more likely to produce such bursts. We planned a Target of Opportunity for when either 4U 1820-30 or 4U 172834 entered into a state where superexpansion bursts are likely to occur. 4U 1820-30 was observed briefly on 2013 July 8, but it was not in the correct state. 4U 1728-34 was observed on 2013 October 1 for $33 \mathrm{ks}$ and on 2013 October 3 for $33 \mathrm{ks}$, and bursts were detected, but they were not 
superexpansion bursts. For a NUSTAR paper on a serendipitous detection of a type I X-ray burst from GRS 1741.9-2853, we refer the reader to Barrière et al. (2014).

\section{Conclusions}

NUSTAR's combination of sensitivity, throughput, and energy resolution is providing significant new results in studies of $\mathrm{X}$-ray binaries. The large dynamic range that NuSTAR provides allows for useful observations of bright (e.g., Cyg X-1) and faint (e.g., Cen X-4) systems. Some of the largest advances over previous satellites can be made with moderate-length observations of sources with intermediate count rates, and IGR HMXBs provide excellent examples. This is illustrated especially by the discovery of a cyclotron line in the SFXT IGR J17544-2619.

\section{References}

Alexander, D. M., et al., 2013, ApJ, 773, 125

Barrière, N. M., et al., 2014, accepted by ApJ, arXiv:1411.4745

Bellm, E. C., et al., 2014, ApJ, 792, 108

Bhalerao, V., et al., 2014, accepted by MNRAS, arXiv:1407.0112

Bozzo, E., Falanga, M., \& Stella, L., 2008, ApJ, 683, 1031

Cackett, E. M., Brown, E. F., Miller, J. M., \& Wijnands, R., 2010, ApJ, 720, 1325

Chakrabarty, D., et al., 2014, ApJ, 797, 92

Coburn, W., Heindl, W. A., Rothschild, R. E., Gruber, D. E., Kreykenbohm, I., Wilms, J., Kretschmar, P., \& Staubert, R., 2002, ApJ, 580, 394

D’Angelo, C. R., Fridriksson, J. K., Messenger, C., \& Patruno, A., 2014, submitted to MNRAS, arXiv: 1410.3760

Duro, R., et al., 2011, A\&A, 533, L3

Fabian, A. C., Rees, M. J., Stella, L., \& White, N. E., 1989, MNRAS, 238, 729

Fabian, A. C., et al., 2012, MNRAS, 424, 217

Fürst, F., et al., 2013, ApJ, 779, 69

Fürst, F., et al., 2014a, ApJ, 784, L40

Fürst, F., et al., 2014b, ApJ, 780, 133

Gotthelf, E. V., et al., 2014, ApJ, 788, 155

Gou, L., et al., 2014, ApJ, 790, 29 
Grefenstette, B. W., et al., 2014, Nature, 506, 339

Harrison, F. A., et al., 2013, ApJ, 770, 103

in't Zand, J. J. M., \& Weinberg, N. N., 2010, A\&A, 520, A81

King, A. L., et al., 2014, ApJ, 784, L2

Lewin, W. H. G., van Paradijs, J., \& Taam, R. E., 1993, Space Science Reviews, 62, 223

Lightman, A. P., \& White, T. R., 1988, ApJ, 335, 57

McClintock, J. E., Shafee, R., Narayan, R., Remillard, R. A., Davis, S. W., \& Li, L.-X., 2006, ApJ, 652,518

Miller, J. M., et al., 2013a, ApJ, 779, L2

Miller, J. M., et al., 2013b, ApJ, 775, L45

Miller, J. M., Pooley, G. G., Fabian, A. C., Nowak, M. A., Reis, R. C., Cackett, E. M., Pottschmidt, K., \& Wilms, J., 2012, ApJ, 757, 11

Miyasaka, H., et al., 2013, ApJ, 775, 65

Mori, K., et al., 2013, ApJ, 770, L23

Natalucci, L., et al., 2014, ApJ, 780, 63

Orosz, J. A., McClintock, J. E., Aufdenberg, J. P., Remillard, R. A., Reid, M. J., Narayan, R., \& Gou, L., 2011, ApJ, 742, 84

Risaliti, G., et al., 2013, Nature, 494, 449

Tendulkar, S. P., et al., 2014, ApJ, 795, 154

Tomsick, J. A., et al., 2014, ApJ, 780, 78

Truemper, J., Pietsch, W., Reppin, C., Voges, W., Staubert, R., \& Kendziorra, E., 1978, ApJ, 219, L105

Yamamoto, T., Mihara, T., Sugizaki, M., Nakajima, M., Makishima, K., \& Sasano, M., 2014, PASJ, 66,59 